\title{
The Data Warehouse Design Problem through a Schema Transformation Method
}

\author{
G. Jagan Naik, V. PC Rao, A. Govardhan
}

\begin{abstract}
An 'operational-data-base' is a common archive of information. The OLTP's are utilized for everyday exchange handling and don't satisfy the out of the requesting assignments of data preparing and information examination. Another structure technique is required to outline OLTP DB's information into MD information display. The MD information display is an objective data reproduction originates from the undertakings, they have measure of information, and however they can't get to it. They utilize a similar pursuit designs everyday and information more often than not will be not set up for a simple questions. The MD show speaks to a measure that relies upon an arrangement of measurements, which gives the setting to the measure it is a choice to exhibit that as hyper $3 D$ square, that are the realistic way and calculated model or by the patterns in a consistent model. Beforehand DWH plan and improvement connected specially appointed approaches. Extending number-of associations are actualizing information distribution centers to fortify their choice emotionally supportive networks. This accompanies the difficulties of the populace and the occasional refresh of information stockrooms. In-this' postulation, a device is presented that-furnishes clients through-highlights toward-make a-stockroom list-and-change structures of the source 'data-base-into-structures' for-the distribution center data-base. This-is profoundly intelligent, simple to-utilize as-well-as shrouds the basic multifaceted 'nature-of-physical-SQL-code' age as-of-its clients. Attributes from source tables can be mapped into new traits in the stockroom data-base tables utilizing total capacities. At that point, applicable information is consequently elated as-of-the' cause-record in-to-the' recently made distribution center. The instrument along these lines incorporates stockroom creation, composition mapping and information populace in-to-a solitary universal-reason-means.
\end{abstract}

The instrument has-been' planned as-a part of-the-structure in-support-of a mechanized information distribution center. Clients of this structure are the data-base chairmen, who will likewise have the capacity to synchronize updates of different replicas-of-the-information'-distribution center. Distribution center pictures that should be refreshed be full disconnected as-well-as 'claims-to-want-to' get into-the-information-store' would currently be able to get to any-of-the-additional picture stockrooms.

The controlling-submission incorporated with it as-well-as system toggles amid data-bases into-the-mode-that-is absolutely straightforward in-to use thus-they don't understand presence of numerous replicas-of-the-information'-stockroom. Essentially, even non-specialized clients can make, populate and refresh information distribution centers with insignificant time and exertion. Finally a DSS will rebuild for making and analyses for concrete queries.

Revised Version Manuscript Received on 16 September, 2019.

* Correspondence Author

G. JaganNaik- Ph.D Scholar, Department of Computer Science \& Engineering, JNTU-Hyderabad, Telangana, India

Dr. V.PC Rao- Professor, Department of Computer Science \& Engineering, MLRIT, Dundigal,Hyderabad, Telangana, India

Dr.A.Govardhan- Professor, Department of Computer Science \& Engineering, JNTU Hyderabad, Telangana, India
Keywords: Information-Warehouse, DW-Structure, DW-Mapping-Production, plan-change, 'Relational-DW', 'DW' Structures'-Follow, MD replica, 'OLAP' and MD-BE.

\section{INTRODUCTION}

Data-base formation is an unpredictable undertaking and includes change numerous parameters as-well-as should subsist possible utilizing every-data-base formation. Resting-on the' off-possibility'-that numerous Information stockrooms are-data-stocks'-that-are stacked with subsets of important information as-of-a-basis data-base. This distribution centers may contain instructive information extricated from operational in-order to-the resource-data-base'. The-'tables'-in stockroom data-bases depend scheduled the-charts-as of-the' basis data-base. Thus, it is fundamental to change arrangements of-the cause data-base in-to arrangements for the stockroom. These days, this-is finished by physically investigating and making 'such-a plan. This-procedure be-together' dull as-well-as tedious. Additionally, clients should be in fact prepared to play out this assignment. There are a couple of different inadequacies in the present framework. In the distribution center construction clients may-add-new ascribes to-tables'; these new properties are the totals of the characteristics of the ace data-base. Therefore, when information is duplicated from the ace data-base to the stockroom data-base, information for these total capacities should be registered at run-time amid refresh, causing more postponement. At the point when this refresh is in advancement, applications getting to the stockroom won't gain admittance to exact information, prompting absence of synchronization. MDBE pursues a traditional methodology, in which the end-client prerequisites are notable already. This methodology profits by the learning caught in the information sources, however controls the plan assignment as per necessities and subsequently, it can labor as well as knob-semantically' inferior information sources. As it were, giving superb end-client necessities, we can manage the procedure from the information they contain, and beat the reality of discarding awful eminence-information sources.

\section{A. Design-Objectives}

These issues shape the premise and the inspiration for this postulation. The proposed postulation instrument is progresses in the direction of giving an interface to acknowledge required data from clients to produce another data-base and makes a vacant information stockroom. For a given source data-base, the proposed device goes for landing at a suitable mapping to make a stockroom structure. After a-planning have be dignified, 
tables-for-the-new-stockroom' are made, at that point, pertinent information is naturally changed as-of the resource-data-base' into-the recently made stockroom. A system has been worked to encourage programmed updates of information stockrooms. This-is-a' planned in-to-a-pathso-as-to' present-tin-be' numerous duplicates of-the' stockroom data-base, 'where-each' duplicate be a picture of the distribution center data-base. Duplicates that should be refreshed are taken disconnected and applications that need to get to the stockroom data-base would now be able to get to any of the other picture distribution centers. The proposed instrument likewise switches relevance - figure-controller, switches between data-bases in-a-path-that is absolutely straightforward to applications with the goal that they don't understand presence of different distribution center data-bases. Accordingly, utilizing the proposed apparatus by end client can specifically make the coveted distribution center blueprint. A noteworthy preferred standpoint in utilizing this proposed device is to mechanize the SQL content age for construction creation and information the executives. The utilization of such a device gives the client more opportunity to structure his pattern more precisely and proficiently instead of building up the code itself.

\section{NOTIONS OF-A COMPUTERIZED-INFORMATION-WAREHOUSE'}

The 'Semi 'Automatic/Routine-SchemaGeneration/Cohort-Mapping' ('ASGM') is an instrument to moves in the direction of giving a-border to acknowledge as-of clients the-necessary order-to produce another data-base as-well-as makes an unfilled information distribution center. For a given source data-base, the instrument goes for landing at a suitable mapping to make a fundamentally related distribution center. Following a planning have-to-be dignified, tables-for-the-novel stockroom are made. At that point, significant information is consequently transported from the source data-base to the recently made distribution center.

\section{A. Information-Warehouses'}

Information warehousing is-a gathering of advances that help the board basic leadership. They can be extensively named choice emotionally supportive networks. Decision help is a methodology expected to isolate information from data and to use this information as an explanation behind essential administration. For example, is a piece of the Web-based Benchmark information motor? It is a choice supporting application that separates information from a distribution center and shows it in visual shape. Leverage of assembling data resembles a visual may prompt location of patterns, connections, special cases and examples in the information, assuming any. Consequently, these perceptions might be a key later on basic leadership forms.

Data dispersion focuses contain a wide collection of data that present a clear picture of business conditions at a lone point in time. Illuminating data is expelled from operational data in the source information base and is changed for end-client basic leadership. The headway of a data dispersion focus fuses enhancement of structures to isolate data from operational systems and the foundation of a stockroom information base structure that gives bosses

versatile access to the data. It is gone for decreasing multifaceted nature and enhancing productivity of information questioning. Data appropriation focuses offer affiliations the ability to collect and store undertaking information in a singular hypothetical undertaking storage facility. Basic data exhibiting methodologies are associated with make connection between individual data parts or data segment social occasions. These affiliations, or models, consistently show up as component relationship mapping.

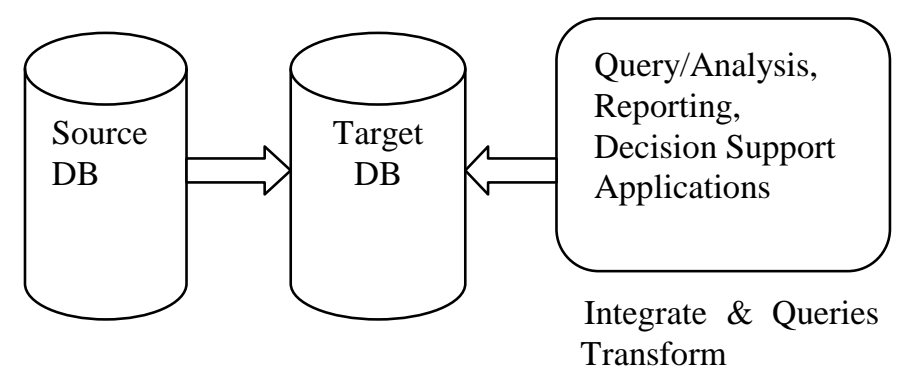

Fig.2.1. Information distribution center engineering

The significant segments in Fig 2.1.are as pursues:

Source information bases - In this postulation, data gathered from-data warehouse is moved to a recently made information distribution center. The ace information base goes about as the source information base.

Data Warehouse (target information base) - The objective information base is the information stockroom that we intend to make and populate. Subset of significant information and synopsis data gathered from-data warehouse exists inside the information distribution center engineering. It is gotten to through questions by work area applications, for example, inquiry and examination, choice help applications and information mining apparatuses.

Decision Support Applications - Decision bolster applications will utilize information from the information distribution center. In this postulation, an application, for example, Chart Visio may go about as a Decision Support Application.

\section{B. 'Structure-Mapping'}

Information warehousing includes mapping sub-sets of relevant data gathered from-data warehouse-to-the goal information base'. The objective information base pattern is structured dependent on the information that is being elated from-the-underlying information base'. Thus, there is a mapping between the structure of the underlying information base' and that of the goal information base'. This mapping is named as Schema Mapping. An information distribution center' is made self-sufficiently, in view of the composition of source information base. Construction Mapping is fundamental way to-change game plans of-the premise data-warehouse in to courses of action for-the-storage facility. This should be possible physically, where clients can physically investigate on making such a mapping, which can be as one exhausting and additionally time-overpowering. This additionally expects clients are on a basic level educated to-do this-errand. 


\section{Computerized Data-Warehouse' \\ Information \\ Refresh \\ In}

Data conveyance focuses contain a wide grouping of data that present a conscious picture of business conditions at a single point in time. Instructive data is expelled from operational data in the source information base, changed for end-customer essential authority and set away in the data dispersion focus. Changing data from the source information base to the data stockroom is a moderate procedure. The tables in the stockroom outline may have new traits, which might be sums of the attributes of the ace information base. In the midst of data trade from the pro information base to the stockroom information base, data for these aggregate limits ought to be figured at runtime, causing more postponement. As of now, the information stockroom will be inaccessible to applications getting to it and these outcomes in absence of synchronization.

\section{Issue-Definition'}

Give us a chance to think about the accompanying situation: Users have a huge information base and need to store a sub-set of information in a distribution center. The procedure associated with doing that is:

- Explore the source information base and choose what information should be spoken to in the distribution center

- Create data storage facility by-change-strictures utilizing information base creation wizard.

- Create-SQL-questions'- to make pattern for the recently made information distribution center.

- Create-SQL-questions'- to move appropriate data as of the premise record into-the data store.

- Periodically deal with the refresh of the information stockroom so changes in the source data-base are reflected in the information distribution center.

- Deal with numerous pictures of the information distribution center with the end goal to guarantee accessibility of information stockroom consistently

- Provide applications with straightforward access to various pictures of information stockroom

This method expects that end-clients know about SQL and commands them to utilize other accessible programming to make an information stockroom.

Programmed refresh of the information distribution center should be actualized utilizing propelled data-base ideas. This is tedious and requires broad specialized help for non-specialized clients. Give us a chance to think about a precedent from an example fabricating industry information base to clarify the issue better. (Note: The assembling business information base is alluded'- to-as-the 'source information base' in this talk.) In this situation, clients need to store execution related data of supplies in an information distribution center. A few errands should be performed to effectively make such an information distribution center.

In the source information base, customers need to pick execution related data set away in the equipment hours table, in qualities - Running Hours, Uptime, MTBF Predicted and
MTBF Required. Furthermore, it is central that the basic key of the Equipment Hours table is a bit of the data stockroom. This is displayed in Figure 2.2. Customers need to make a data circulation focus by tuning parameters, using Oracle's information base creation wizard. Customers need to make the Equipment Hours table in the data conveyance focus, with simply the required characteristics and exchange relating information.

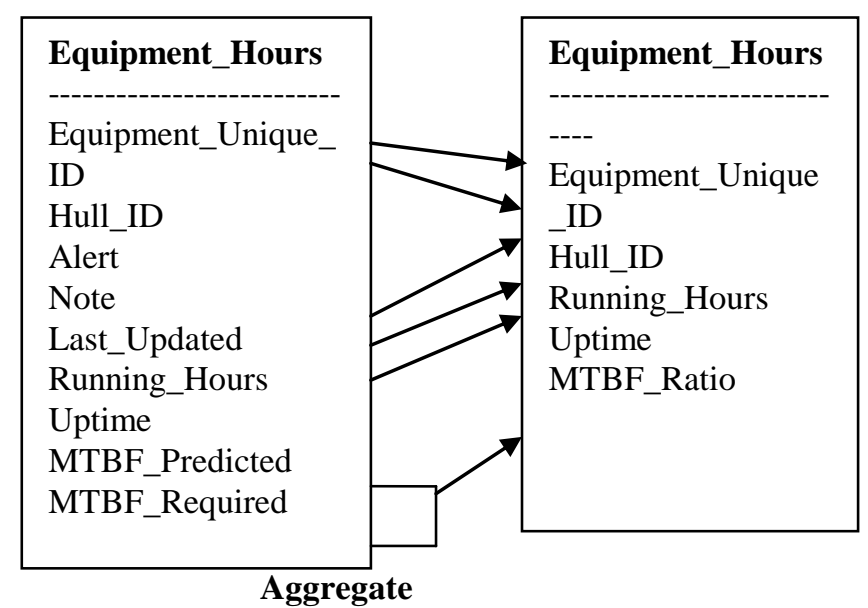

Table Structure in Source Schema Table Structure in Target Schema

Figure.2.2. Property-Selection from-Source-Table

It must be noticed that the Equipment Hours table has a few referential trustworthiness imperatives, because of which all the parent tables should be a piece of the information distribution center construction. Parent tables of Equipment Hours table are introduced in Figure 2.3.

\begin{tabular}{|c|c|c|}
\hline Equipment_ Hours & & Equipment Hours \\
\hline Equipment & & \\
\hline $\begin{array}{l}\text { Manufacturer Info } \\
\text { Shins }\end{array}$ & & $\begin{array}{l}\text { Equipment } \\
\text { Manufacturer Info }\end{array}$ \\
\hline Ships System & Table & Ships \\
\hline Model No Info & Selecte & Ships System \\
\hline Next Higher & & Model_No_Info \\
\hline _Assembly Vessel_ & & Next_Higher_ \\
\hline Group & & Assembly \\
\hline Vessel_roup_Vessel & & \\
\hline
\end{tabular}

All referential Tables

Automatically selected

Source DB

Target DB

Figure2.3. Tables-Selection-from Source-Data-base

Clients may likewise need to incorporate another quality 'MTBF Ratio' that figures the extent as a sum of MTBF Predicted and MTBF Required. In this model, we have considered mapping only a solitary table from the source information base to the data stockroom. The issue creates as the amount of tables and credits to be mapped increments. 


\section{E. An-Answer For-The Issue:}

As an answer for the issues referenced over, the proposed instrument that produces another information stockroom, performs blueprint mapping and constructs a structure for programmed refresh of the information distribution center. The proposed tool is to allow users to select, extract, clean, and convert information-from-basis-scheme structures into consistent target warehouse data structures. Also, the information from the source data-base is populated into the objective data-base. The information stockroom can be populated on a recurrence that addresses the association's issues. The instrument explores clients in a grouping of intuitive advances and acknowledges the parameters to make another information stockroom. For-a-known basis-data-base, 'the-instrument assists clients 'in' touching base on a fitting planning-to' makes 'a-basically related stockroom. Following a-planning 'has-been' striated, tables-are-made. At that point, significant information is consequently changed from the source data-base to the recently made distribution center. To-allow automatic inform of-the-store'-record, a setup has been built that manages the periodic update of the warehouse. Applications right-of-entry-the information store through an interface that provides a simple-to-use API. Users may create manifold-images' of-the information-storehouse using the tool. The support to update all the images is provided in the framework.

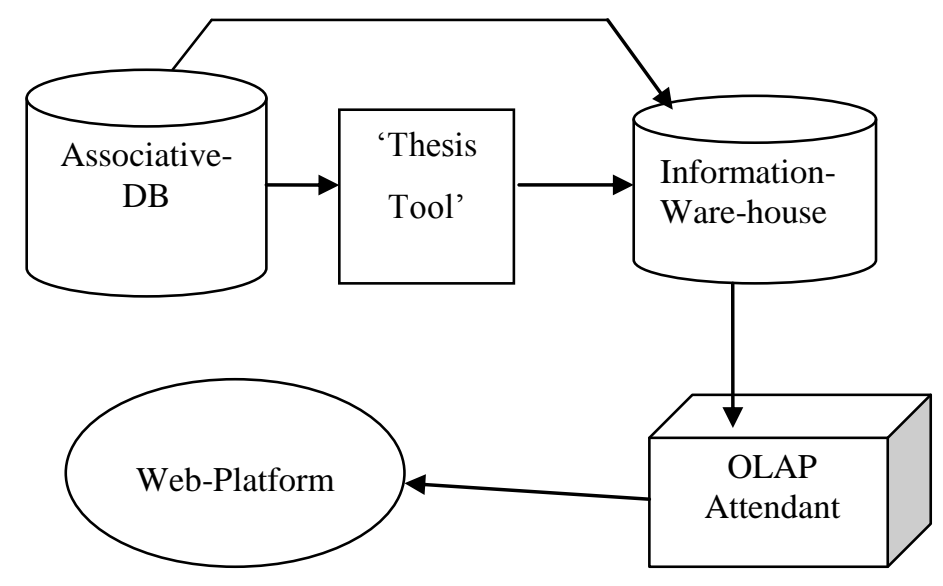

Fig 2.5 System Architecture

\section{SYSTEM-'DESIGN' SUMMARY}

Framework engineering is an essential segment of an application structure. Designing decodes the reliable arrangement of the application to a structure that portrays the joint effort of components in the system. The proposed framework plan in this theory all together best determination the issue of the MD information base is the person who is appeared in the figure 2.5. This framework is made by different portions: a social information base, the proposition mechanical assembly, a data stockroom, an OLAP server and a web circumstance. The Relational information base is an information base secured in an information base chief.

- This information base chairman must be a PostgreSQ manager. The proposition gadget is a program worked in JAVA.
- This instrument needs to interface with the social information base administrator with the true objective to scrutinize an information base. It needs to interface with the data stockroom director with the true objective to make another information base and present data. The proposition gadget needs to access to the OLAP server list, with the true objective to store the OLAP mapping and the MDX question.

- The Data circulation focus is the information base that the recommendation gadget has made. It is store in a social information base chief. This information base chief must be a PostgreSQL manager.

- The OLAP server is an open source server has a place with the stage business knowledge, which gives an aggregate suite to examinations business data. This OLAP server is a module that continues running into a Tomcat web server.

- The Web condition is a passage to the data through http channel.

\section{INFORMATION-SERVER-'CREATION'}

Information base creation is a confusing errand and incorporates tuning various parameters. This area depicts how SagaMap gives a graphical interface to recognize the fundamental parameters to create content records, which can be executed from the request induce to make another information base. One of the general objectives of this venture it to have the capacity to make and populate information bases for information distribution centers. This includes making a clear information base, in which information might be filled in. In this section, we examine how our instrument helps towards achieving the initial step of the objective. UI configuration depicts how contents are produced and executed in-to-make a-novel distribution center information base.

\section{SELF-LOADER-GENERATION OF WARE-'HOUSE' 'SCHEMA'}

As expressed already, the-goal of'- this ventures it to have the capacity to make and populate information bases for information stockrooms. This likewise includes making an information stockroom pattern and stacking the distribution center by sub-sets'- of appropriate data as-of the-premise'record. The proposed apparatus to enable clients to choose, concentrates, clean, and changes over data as-of-premise conspire structures into reliable target stockroom information structures. Likewise, the-data as-of the-premise record is populated into the objective information base. The data store-have the capacity to' be populated on a recurrence that addresses the association's issues. An information distribution center depends absolutely on its capacity draw data from over the association. The proposed apparatus gives clients the capacity to associate with any source information base to draw the required data. Data is drawn into the stockroom by solidifying and purging information before populating the distribution center information base. This is done consequently after clients settle the objective information base pattern and the mapping with the source information base diagram. 
Information warehousing includes mapping subsets of appropriate data from the source information base to the goal information base. The objective information base outline is planned dependent on the information that is being transported from the source information base. From this time forward, there is a mapping between the structure of-the-premise information base and the objective information base. This mapping is named as Schema Mapping. For a given source information base, the mechanical assembly enables customers in meeting up at a fitting mapping to make a semantically related appropriation to focus. After a mapping has been formalized, another stockroom is made. By then, pertinent data is normally transported from the source information base to the as of late made stockroom. Each mapping made for a source and target information base is put away in XML documents. This guarantees clients can take off further upgrades to the mapping by stacking them at a later time.

This apparatus gives clients a graphical interface to perform Schema Mapping.

- Select source information base: Specify the ace information base for which the circulation focus ought to be made

- Select tables: Users may choose just significant tables from the source information base to be a bit of the goal information base. Figure 5.2. Presents a mapping outline to demonstrate a precedent.

- Enforce referential respectability: If youngster tables are chosen to be a bit of the objective information base, the relating expert tables ought to be chosen as well. The ace tables are inside registered by the apparatus and are chosen naturally.

\section{CONCLUSION AND FUTURE WORK}

The proposed thesis tool assumes a fundamental job in providing support for automated data warehouses [1]. It is simple to use, highly interactive and provides an easy means to creating another information distribution center. It likewise goes about as a dependable device to rapidly investigate composition of the source data-base with the end goal to create construction for the information distribution center. The proposition apparatus fundamental complex components from its clients, aside from where it is completely suitable and important to uncover them. As a result, even non-specialized clients can make, populate and refresh information distribution centers with negligible time and exertion. Qualities from resource board canister-exist mapped in to novel characteristics 'in-the' distribution center data-base structures utilizing total capacities. At that point, pertinent information is consequently changed as-of the resource-data-base' to the recently made stockroom. The instrument in this manner coordinates stockroom creation, construction mapping and information populace into a solitary universally useful device. This device has been planned as a segment of a structure, whose clients are Data-base Administrators. They will likewise have the capacity to synchronize updates of numerous replicas-of-the-data' distribution center. Distribution center pictures that should be refreshed full disconnected as-well-as requests 'so-as-to' require toward-get into-the-information-store would now be able to get to any of

the other picture stockrooms. The' 'Image '-' Switcher' incorporated with its-structure switches between data-bases into-the-path-that' is absolutely straightforward in-to requests thus-they' don't understand presence of different replicas-of-the-data distribution center. It additionally guarantees that continuous exchanges on a specific data-base be-not' hindered while the-information-base be booked to be taken disconnected. The proposition device is framework free. It provides focal points, for example, convey ability as-well-as wide application. This device can get to any information base with negligible exertion since there is no hard coding of data in the claim.

\section{RESULTS:}

\section{Algorithm Multidimensional_Modeling Input:}

$\mathrm{T}:\{\mathrm{t} j\}$ set of tables of the relational model

$\mathrm{R}:\{$ ( ai , aj ) $\}$ set of foreign key relations

Output:

$\mathrm{FT}=(\{\mathrm{g}\},\{\mathrm{m}\})$

$\mathrm{D}=\{\mathrm{d}\}$

$\mathrm{AS}=\{\mathrm{a}\}$

\section{Variables used in the algorithm}

G: variable used to store a grain attribute

$\mathrm{m}$ : variable used to store the measure attribute.

P: $\{$ pi $\}$ : set of path in construction. They start in the grain attribute $g$.

p: a path.

\section{Begin:}

More Grains: Boolean: = true;

$\mathrm{AS}=\{\}$

$\mathrm{m}:=$

select_measure_attribute_from_relational_model();

// Dimensions

While (moreGrains) loop

$\mathrm{g}:=$ select _ grain _ attribute _ from _ relational _ model();

// $P:\{p\}$ set of path from the measure grain attribute to the measure attribute

$\mathrm{P}=$ make Paths Grain to Measure (T, R, $g, \mathrm{~m})$;

For all p Î P loop

// d: new dimension

$\mathrm{d}:=$ make Dimension(T, R, AS, g);

$\mathrm{D}:=\mathrm{D} \grave{\mathrm{E}}\{\mathrm{d}\}$

$\mathrm{P}:=\mathrm{P} \grave{\mathrm{E}}\{\mathrm{p}\}$

\section{End loop;}

More Grain: = user_answer ();

End loop;

// Fact Table

For all $\mathrm{d} \in \mathrm{D}$ loop

$\mathrm{g}^{\prime}:=$ grain _ attribute of $(\mathrm{d})$;

FT: $=\left(\{\mathrm{g}\} \overline{\mathrm{E}}\left\{\mathrm{g}^{\prime}\right\},\{\mathrm{m}\}\right)$

\section{End loop}

\section{End algorithm;}




\section{REFERENCES}

1. LGI Systems Incorporated: A Definition of Data Warehousing, http://www.dwinfocenter.org/defined.html.

2. LGI Systems Incorporated: Maintenance issues for Data Warehousing Systems, http://www.dwinfocenter.org/maintain.html.

3. A.Gittleman: Advanced Java Internet Applications, 2nd Edition, Scott Jones Inc, 2002

4. J.Hunter and B. McLaughlin: Easy Java-XML integration with JDOM,http://www.javaworld.com/javaworld/jw-05-2000/jw-0518-jd om.html, May 2000

5. S. Chaudhuri and U. Dayal, An overview of data warehousing and OLAP technology, ACM SIGMOD Record, Vol. 26 (1997), pp. 65-74.

6. A. Cuzzocrea, D. Sacca and P. Serafino, A hierarchy driven compression technique for advanced OLAP visualization of $M D$ data cubes, in Proc. of 8th Int'l Conf. on Data Warehousing and Knowledge Discovery (DaWak), (Springer Verlag ,2006), pp. 106-119.

7. A. Cuzzocrea, D. Sacca and P. Serafino, A hierarchy driven compression technique for advanced OLAP visualization of $M D$ data cubes, in Proc. of 8th Int'l Conf. on Data Warehousing and Knowledge Discovery (DaWak), (Springer Verlag ,2006), pp. 106-119.

8. V. Peralta, A. Marotta and R. Ruggia, "Towards the automation of data warehouse design,” Technical Report TR-03-09, InCo, Universidad de la República, Montevideo, Uruguay, June 2003.

9. V. Mani Sarma, Prof.Premchand "MD Context -Dependent information Delivery on the Web" ISSN 0973-6107, Volume 4, Advanced in Computational Sciences and Technology (ACST) November 2 (2011), pp.131-143.

10. N. Huyn. "Efficient View Self-Maintenance". Proceeding of ACM Workshop, Montreal, Canada. 1996.

11. Mr. G. Jagan Naik, (gjnaik1106@gmail.com) Dr. A.Govardhan, Dr. P C Rao, International Journal of Research Volume 7, Issue X, October/2018ISSN NO:2236-6124.

12. Mr. .G. Jagan Naik,( ginaik1106@ gmail.com), Dr. A.Govardhan, Dr. P C Rao, IJRECE Vol. 6 Issue 1 Jan.-March. 2018 ISSN: 2393-9028 (Print) | ISSN: 2348-2281 (Online)

\section{AUTHORS PROFILE}

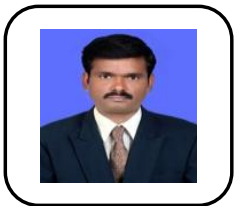

Mr.G. JAGAN NAIK, B.E, M.Tech, Ph.D Scholar, Life member of ISTE --LM 56805 (MISTE), Life membership of International Association of computer science and information technology (IACSIT)-80344902, Life member of International association of engineer (IAENG)-125526, Faculty Development Programs \& Workshops are : $9 \&$ 4,Huge Teaching Experience in Engineering Colleges

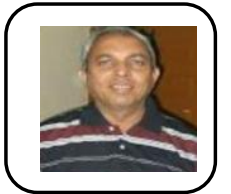

Dr.V.PC Rao, Professor, B.E, M.Tech, Ph.D recent publications Three papers on 1."Region Based Medical Image Compression With Binary Plane Coding" ARPN Journal of E and AS ISSN 1819-6608 2018, 2. “A Kernel Based Apriori Algorithm for Sequential Pattern Mining” International Journal of Research ISSN: 2348-6848 2017 \& 3. "Sequential Pattern, Rule Growth Mining Implication for Knowledge Representation, Identification of Balanced Relationships between Present and Next Stage Medications in Medical Analysis” JARDC ISSN 1943-023X 2018.

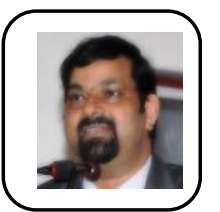

Dr. A Govardhan, B.E.(CSE), M.Tech(CS), Ph.D(CSE), SMCSI, MACM, MIEEE, MIAENG, MWASET, LMISTE, MFSF, MTDWI, SMIACSIT, Computer Science \& Engineering, He believes that following three things are necessary to make every person great and every nation great:

Conviction of the Powers of good, Absence of Jealousy and Suspicion, Helping all who are trying to be good and do good, Dr.A.Govardhan is presently a Professor of Computer Science \& Engineering, Rector and Executive Council Member, Jawaharlal Nehru Technological University Hyderabad (JNTUH). 